\title{
Análise correlativa entre medidas esqueléticas verticais em indivíduos com oclusão excelente e má oclusão
}

Maridélia Machado Mateus Damico*, Fernanda Catharino Meneses Franco**,

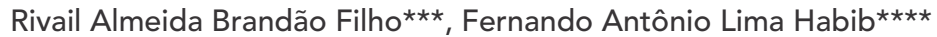

\section{Resumo}

Introdução: avaliar as proporções verticais faciais é um dos parâmetros mais importantes a ser utilizado pelo ortodontista no planejamento da terapia apropriada. Porém, não é raro que, quando se avaliam medidas isoladamente, tenha-se uma idéia errônea do que a face do paciente apresenta em termos de padrão vertical. Objetivos: o presente trabalho realizou diferentes correlações entre a ângulo do plano mandibular (GoGn-SN), o índice de altura facial (IAF) e a sobremordida, avaliando a resposta dessas correlações quanto ao padrão citado na literatura, além de identificar possíveis fatores que, eventualmente, levassem essas medidas a um comportamento fora desse padrão. Metodologia: o estudo foi realizado em radiografias cefalométricas de perfil entre dois grupos de indivíduos brasileiros - um portador de oclusão excelente e outro de má oclusão - atendidos no Curso de Especialização em Ortodontia e Ortopedia Facial da Faculdade de Odontologia da Universidade Federal da Bahia. Resultados: as medidas esqueléticas GoGn-SN e IAF apresentaram forte correlação entre si. A sobremordida, entretanto, não apresentou correlação com essas medidas no grupo com oclusão excelente e demonstrou fraca correlação naquele com má oclusão. Uma correlação coerente com o padrão citado na literatura foi encontrada em 35\% e 31\% dos indivíduos dos grupos com oclusão excelente e má oclusão, respectivamente. Os indivíduos restantes apresentaram variações na altura dentoalveolar dos incisivos, compensando as displasias verticais. No grupo com oclusão excelente, esse fator se somou às compensações na região de molares. Conclusões: os resultados encontrados indicam que a maior parte dos grupos apresentou um comportamento diferente do padrão citado na literatura, quando as três medidas foram correlacionadas simultaneamente.

Palavras-chave: Ângulo do plano mandibular. Índice de altura facial. Trespasse dentário vertical. Dimensões verticais da face.

* Especialista em Ortodontia e Ortopedia Facial - UFBA - Salvador/BA.

** Mestre em Ortodontia - UFRJ. Professora do curso de Especialização em Ortodontia e Ortopedia Facial / Faculdade de Odonto-

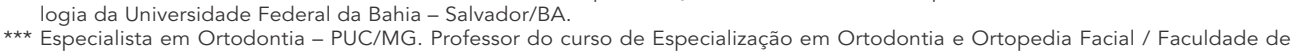
Odontologia da Universidade Federal da Bahia - Salvador/BA.

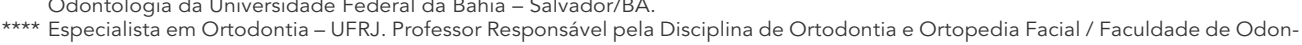
tologia da Universidade Federal da Bahia - Salvador/BA. 


\section{INTRODUÇÃO}

As análises cefalométricas fornecem detalhes imprescindiveis ao correto diagnóstico ortodôntico. Entretanto, as medidas interpretadas isoladamente nem sempre representam o verdadeiro padrão facial do paciente, sendo, por vezes, necessário correlacioná-las. Além disso, os valores-padrão dessas análises se aplicam melhor a indivíduos com características semelhantes às da amostra estudada.

Neste sentido, ressalta-se o valor do ângulo GoGn-SN, que pode sofrer influência de inclinações na base do crânio. Segundo Anderson, Popovich $^{1}$; Björk, Skeiller ${ }^{8}$; Jacobson ${ }^{18}$ e Nielsen ${ }^{31}$, variações anormais na configuração e no comportamento da base do crânio podem resultar em desarmonias dentofaciais severas. Uma inclinação inferior muito acentuada na sela túrcica pode posicionar a região posterior da face em um nível muito baixo, resultando em uma menor inclinação do plano mandibular. Contrariamente, uma base craniana anterior relativamente horizontal pode posicionar a face posterior em um nível muito alto, tendo como efeito um plano mandibular anormalmente íngreme e retrusão mandibular ${ }^{10}$.

Outro aspecto que também pode influenciar o ângulo GoGn-SN é a presença de um crescimento vertical excessivo dos processos alveolares posteriores. Tal crescimento atua como coadjuvante na rotação póstero-inferior da mandíbula e, portanto, no aumento do GoGn-SN. Contrariamente, uma deficiência na altura dessa área propicia uma rotação ântero-superior mandibular e, portanto, está freqüentemente relacionada a indivíduos de face

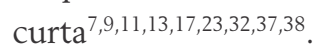

Para Horn ${ }^{15}$, o índice de altura facial (IAF) oferece uma indicação mais precisa do posicionamento vertical da mandíbula, pois capacita o clínico a monitorar, cuidadosamente, não só o comportamento mandibular, mas também as relações esqueléticas maxilomandibulares. O IAF é o produto da razão entre as alturas faciais posterior (AFP) e anterior (AFA). Primariamente, os fatores que determinam o aumento na AFA são o crescimen- to vertical dentoalveolar nas regiões posteriores $\mathrm{da}$ maxila e mandíbula e o deslocamento inferior da maxila, decorrente do crescimento sutural. A AFP, por outro lado, é determinada pelo crescimento vertical condilar. Este índice tem valor médio de 0,70 ; variações entre 0,65 e 0,75 são consideradas dentro da faixa de normalidade ${ }^{15,16,31,35}$.

Se o IAF aumenta significa que o crescimento do ramo é proporcionalmente maior que o crescimento vertical da porção ântero-inferior da face, e a tendência é o aprofundamento da mordida. Se o IAF diminui, o crescimento vertical ânteroinferior facial é proporcionalmente maior que o crescimento do ramo, e a tendência é a abertura da mordida ${ }^{15,16,28}$.

Tanto o grau de inclinação do plano mandibular (GoGn-SN) quanto o IAF estão relacionados com o nível de trespasse dentário vertical, pois este depende largamente da relação entre a quantidade de crescimento dentoalveolar vertical dos dentes posteriores e o crescimento dos côndilos mandibulares ${ }^{31}$.

Para Tsai ${ }^{39}$, é a relação entre o crescimento condilar e o crescimento vertical relativo dos processos alveolares - não só na região posterior dos maxilares, mas também na anterior - que controla a quantidade de sobremordida. No entanto, pouca importância é dada ao crescimento dentoalveolar na região dos incisivos, cuja natureza compensatória evita, muitas vezes, o surgimento de uma mordida aberta ou sobremordida profunda, mesmo em casos de pacientes com características inerentes às mesmas ${ }^{5,14,22,30,36}$.

Ao se considerar a variação vista na base do crânio, e em cada estrutura que compõe o complexo dentofacial, verifica-se que não pode haver qualquer combinação rígida de medidas associadas a qualquer tipo de oclusão. Os ajustes e combinações são infinitos e é a integração destas variáveis que determina a harmonia ou desarmonia da face ${ }^{10,25}$. Portanto, um único parâmetro não é suficiente para identificar acuradamente um dado tipo facial ${ }^{24,29}$. 
Considerando-se haver um consenso entre a maioria dos autores - de que as discrepâncias verticais estão entre os maiores desafios de tratamento para o ortodontista - a correlação entre medidas faciais verticais poderia contribuir sobremaneira para o diagnóstico ortodôntico, propiciando maior riqueza de detalhes e, conseqüentemente, um planejamento mecânico mais adequado às características individuais do paciente.

Pensando assim, este estudo foi desenvolvido com o objetivo de avaliar, cefalometricamente, em indivíduos brasileiros portadores de oclusão excelente e má oclusão, a existência de correlação entre as medidas verticais: ângulo do plano mandibular e índice de altura facial, ângulo do plano mandibular e nível de sobremordida, e índice de altura facial e nível de sobremordida.

Além disso, foi avaliado, em ambos os grupos de indivíduos, o comportamento simultâneo dessas três medidas, procurando identificar os possíveis fatores que, eventualmente, pudessem levar estas a uma correlação fora do padrão citado na literatura. Isto implica na oportunidade de discutir o comportamento dessas medidas na população brasileira, diante da grande escassez de trabalhos dessa natureza, fornecendo parâmetros de comparação para o diagnóstico e plano de tratamento ortodônticos.

\section{MATERIAL E MÉTODOS}

Para este estudo, foram utilizadas radiografias cefalométricas em norma lateral de indivíduos brasileiros, não submetidos a tratamento ortodôntico, com todos os dentes permanentes completamente irrompidos, exceto os terceiros molares.

A amostra foi dividida em dois grupos, a saber: o primeiro foi composto por 20 radiografias de indivíduos portadores de oclusão excelente, obtidas aleatoriamente, com média de idade de 26 anos e 7 meses, sendo 14 do gênero feminino e 6 do gênero masculino; e o segundo, de radiografias de 64 indivíduos portadores de diferentes tipos de má oclusão, com idade média de 17 anos e 7 meses, sendo 41 do gênero feminino e 23 do gênero mas- culino, selecionadas do arquivo de pacientes da clínica do Curso de Especialização em Ortodontia e Ortopedia Facial da Faculdade de Odontologia da Universidade Federal da Bahia.

Os cefalogramas foram traçados por um único operador, obtendo-se as medidas cefalométricas ângulo do plano mandibular (GoGn-SN), índice de altura facial (IAF) e sobremordida (Fig. 1). Para se investigar o tamanho mínimo necessário da amostra foi utilizado o índice Kappa, o qual aferiu o tamanho da amostra, indicando sua compatibilidade com a análise desejada $(\mathrm{k}=0,97)$. Também se utilizou o índice Kappa para investigar a existência de erros nos traçados, devido a falhas do examinador. Para tanto, as radiografias foram traçadas e medidas duas vezes, com intervalo mínimo de 20 dias. Os resultados obtidos indicaram uma fidedignidade aceitável dos traçados, pois todos apresentaram excelente chance de concordância.

Para se investigar o sentido de concordância entre os pares de características, aplicaram-se os coeficientes de correlação de Pearson e Spearman. Ambos os coeficientes medem a correlação entre pares de variáveis: GoGn-SN e IAF, GoGn-SN e sobremordida e IAF e sobremordida. Porém, enquanto o de Pearson utiliza os valores absolutos das mesmas para medir o grau de associação linear entre pares de variáveis, o coeficiente de Spearman utiliza variáveis qualitativas ordinais, pois quantifica o grau de associação entre essas variáveis.

A avaliação do comportamento das três medidas, ao serem correlacionadas simultaneamente, foi realizada analisando-se cada traçado individualmente. $\mathrm{O}$ indivíduo era considerado dentro do padrão relatado na literatura quando a seguinte situação era observada: GoGn-SN entre 27 e 35 graus, IAF entre 0,65 e 0,75 , e sobremordida correspondente entre $10 \%$ e $40 \%$ de trespasse do incisivo inferior na face lingual do incisivo superior. Depois de realizada esta análise, foi calculada a percentagem de indivíduos que apresentava o comportamento das medidas coerente e não-coerente com a literatura. 


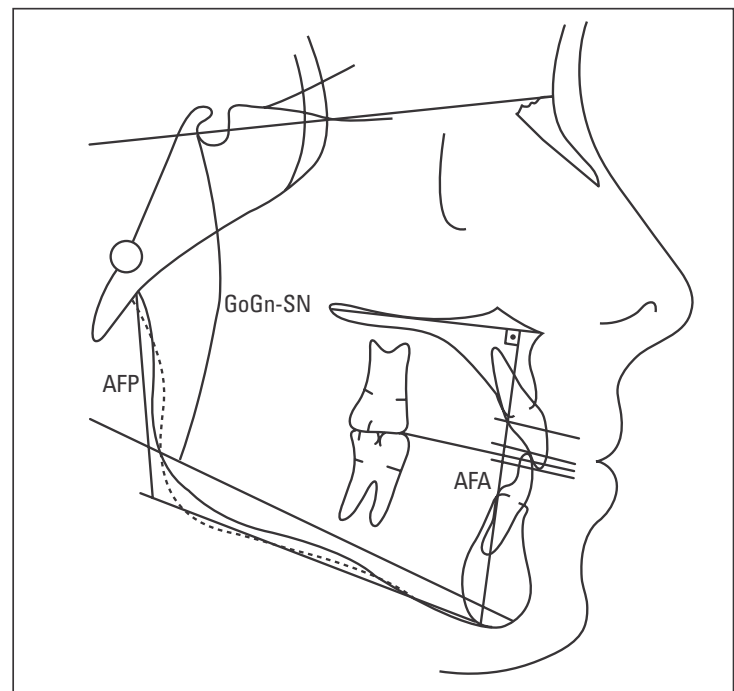

FIGURA 1 - Cefalograma com a localização do ângulo do plano mandibular (GoGn-SN), índice de altura facial (IAF = AFP/AFA) e sobremordida.

A análise dos fatores que poderiam exercer influência sobre o comportamento das medidas GoGn-SN, IAF e sobremordida foi realizada avaliando-se seis características pertinentes, conforme pode-se observar na figura 2:

1) alturas dentoalveolares dos primeiros molares inferiores (MI, valor médio: $28 \pm 2,01 \mathrm{~mm})^{19}$;

2) alturas dentoalveolares dos primeiros molares superiores (MS, valor médio: $20 \pm 1,81 \mathrm{~mm})^{19}$;

3) alturas dentoalveolares dos incisivos centrais inferiores (II, valor médio: $39 \pm 2,93 \mathrm{~mm})^{19}$ e superiores (IS, valor médio: $28 \pm 2,12 \mathrm{~mm})^{19}$;

4) discrepância intermaxilar ântero-posterior

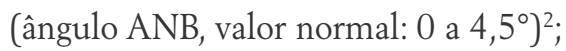

5) ângulo formado entre a linha $\mathrm{SN}$ e o plano horizontal de Frankfurt (SN-PF; valor médio: 5 a $\left.7^{\circ}\right)^{27}$;

6) altura do ramo mandibular (Ar-Go, valor médio: $52 \pm 6 \mathrm{~mm})^{12}$, para auxiliar na constatação da presença de compensações nas medidas já mencionadas.

\section{RESULTADOS E DISCUSSÃO}

No grupo de indivíduos portadores de oclusão

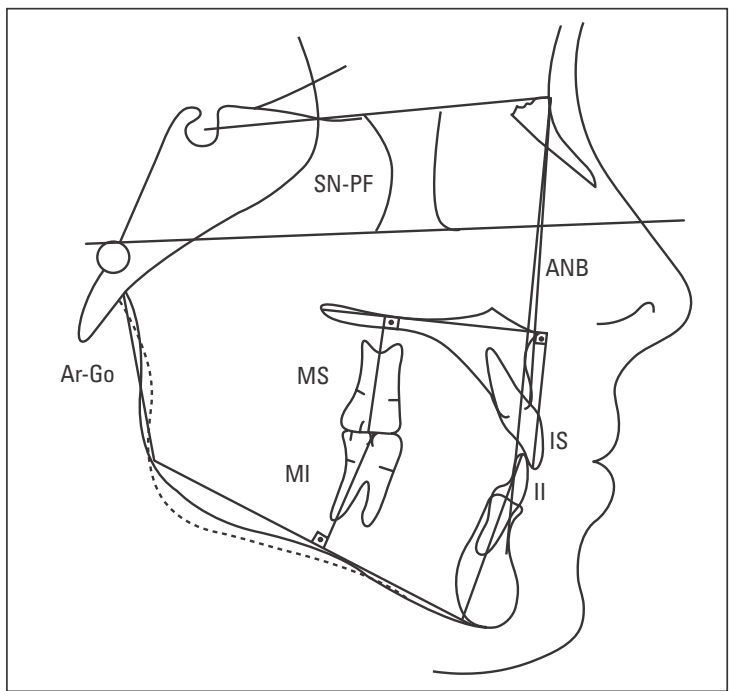

FIGURA 2 - Cefalograma com a localização das alturas dentoalveolares dos molares superiores e inferiores (MS e MI), incisivos superiores e inferiores (IS e II), do ramo mandibular (Ar-Go), do ângulo ANB e do ângulo formado entre a linha sela-násio e o plano horizontal de Frankfurt (SN-PF).

excelente, conforme tabela 1 , foi observada ausência de correlação significativa da sobremordida com GoGn-SN, bem como com o IAF, e uma forte correlação negativa entre as medidas esqueléticas GoGn-SN e IAF.

Independente dos valores de GoGn-SN e IAF, a sobremordida encontrava-se sempre dentro dos valores de normalidade, visto ser esta uma condição

Tabela 1 - Coeficientes de correlação de Pearson e Spearman para as medidas dos indivíduos portadores de oclusão excelente.

\begin{tabular}{cccc}
\hline $\begin{array}{c}\text { correlação de } \\
\text { Pearson }\end{array}$ & GoGn-SN & IAF & sobremordida \\
\hline GoGn-SN & 1,000 & $-0,78716^{* *}$ & 0,10510 \\
IAF & $-0,78716^{* *}$ & 1,000 & $-0,09425$ \\
sobremordida & 0,10510 & $-0,09425$ & 1,000 \\
\hline $\begin{array}{c}\text { correlação de } \\
\text { Spearman }\end{array}$ & GoGn-SN & IAF & sobremordida \\
\hline GoGn-SN & 1,000 & $-0,73482^{* *}$ & 0,08039 \\
IAF & $-0,73482^{* *}$ & 1,000 & $-0,08012$ \\
sobremordida & 0,08039 & $-0,08012$ & 1,000 \\
\hline ** Significância ao nível de $1 \%$. & &
\end{tabular}




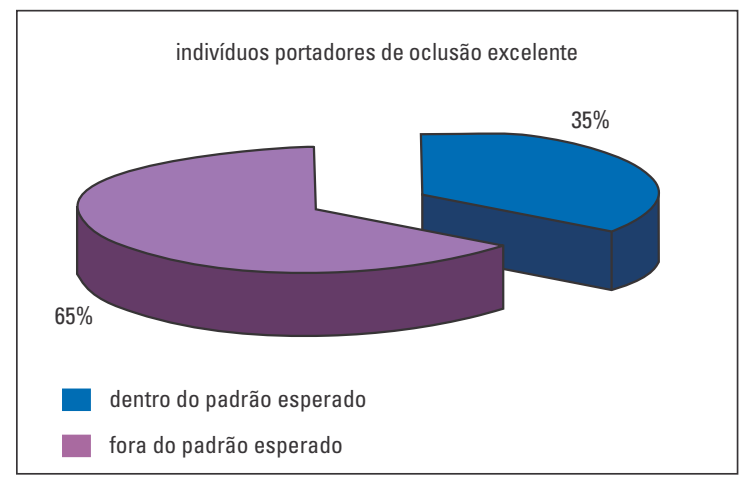

GRÁFICO 1 - Representação gráfica da porcentagem de indivíduos portadores de oclusão excelente cujo comportamento das medidas se apresentou dentro e fora do padrão citado na literatura.

para que o indivíduo pertencesse à amostra. Em relação ao GoGn-SN, tanto os indivíduos com plano mandibular diminuído quanto aumentado tinham sobremordida normal. Portanto, a ausência de correlação significativa entre GoGn-SN e sobremordida era, de certa forma, um resultado esperado. Esses resultados são confirmados por Beckmann et al. ${ }^{4,5}$ e Betzenberger et al. ${ }^{6}$, que afirmaram não haver correlação entre o ângulo do plano mandibular e o nível de sobremordida, através da qual se possa basear o diagnóstico e o plano de tratamento.

$\mathrm{O}$ mesmo raciocínio citado acima com relação ao GoGn-SN pode ser aplicado para o IAF, e está de acordo com Proffit ${ }^{34}$, que afirma que, se o ângulo formado entre a maxila e a mandibula é baixo, há uma relação que predispõe ao trespasse dentário vertical excessivo, independente da presença do mesmo. É corroborado, também, por Beckmann et al. ${ }^{4}$, ao advogarem que o nível de sobremordida independe da divergência entre os maxilares.

Quando foram correlacionados os valores esqueléticos de GoGn-SN com IAF, houve uma tendência de comportamento definida, ou seja, quanto maior era o GoGn-SN menor era o IAF, e vice-versa. Estes resultados suportam as afirmações de Horn ${ }^{15,16}$, Merrifield et al. ${ }^{28}$ e Tsang et al. ${ }^{40}$ Isso ocorreu porque ambas as medidas apresentavam variação dos valores de normalidade, apesar

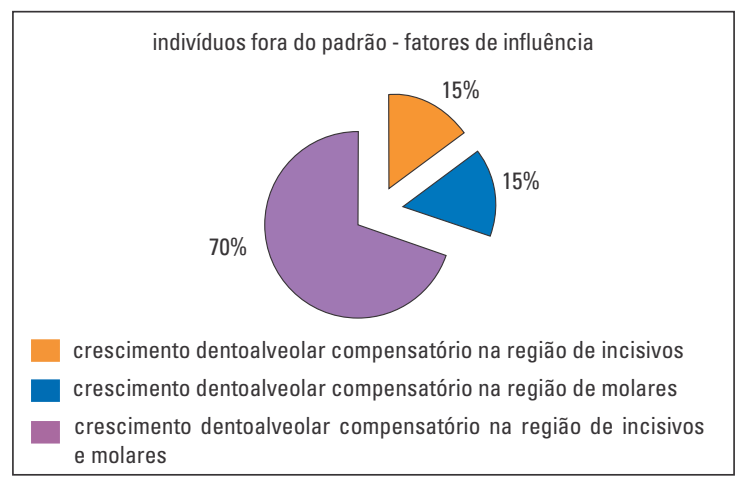

GRÁFICO 2 - Representação gráfica da porcentagem de cada fator que influenciou para que os indivíduos portadores de oclusão excelente apresentassem 0 comportamento das medidas fora do padrão citado na literatura.

desta amostra ser portadora de oclusão excelente e perfil facial agradável.

Ao se analisar, no gráfico 1, o comportamento da correlação simultânea das três medidas, em cada indivíduo portador de oclusão excelente, observou-se que, em 35\% deles, as medidas GoGn$\mathrm{SN}$, IAF e sobremordida apresentaram, entre si, um comportamento dentro do padrão citado na literatura, ou seja, diante da sobremordida adequada, o GoGn-SN e o IAF também se mostravam dentro da faixa de normalidade. O restante dos indivíduos (65\%) não apresentou coerência entre os valores dessas medidas. Assim, mesmo possuindo sobremordida adequada, houve alguma variação do normal em relação aos valores do GoGn-SN e/ou do IAF. Portanto, na maioria dos indivíduos portadores de oclusão excelente, a correlação simultânea das medidas GoGn-SN, IAF e sobremordida apresentou um comportamento fora do padrão descrito na literatura.

No gráfico 2 observa-se que, dos possíveis fatores que levaram os indivíduos a apresentarem o comportamento das medidas fora desse padrão, $70 \%$ deveram-se ao crescimento dentoalveolar compensatório na região de incisivos e molares, $15 \%$ ao crescimento dentoalveolar compensatório apenas na região de molares, assim como $15 \%$ apenas na região de incisivos.

Constata-se, portanto, que, mesmo se tratando 
de uma amostra com características oclusais e faciais satisfatórias, houve, na maioria dos casos, um certo grau de displasia esquelética vertical (GoGnSN e/ou IAF aumentado ou diminuído), porém em intensidade passível de ser compensada pela altura dos processos alveolares de molares e/ou incisivos, normalizando, assim, a sobremordida. O mecanismo compensatório na região dos incisivos foi citado na literatura por Beckmann et al.5; Haralabakis et al. ${ }^{14}$; Karlsen ${ }^{22}$; Naumann et al. ${ }^{30}$; Nielsen ${ }^{31}$; Sinclair, Little ${ }^{36}$ e Tsai ${ }^{39}$. As diferenças nas alturas dos molares nos diversos tipos faciais também já foram constatadas por Haralabakis et al. ${ }^{14}$; Janson, Metaxas, Woodside ${ }^{19}$ e Karlsen ${ }^{20}$, dentre outros.

No grupo de indivíduos portadores de má oclusão, os valores da tabela 2 indicam que existe uma forte correlação negativa entre GoGn-SN e IAF, uma fraca correlação negativa entre GoGn-SN e o nível de sobremordida, e uma fraca correlação positiva entre IAF e o nível de sobremordida.

Os achados da literatura sugerem que há uma tendência de indivíduos com GoGn-SN aumentado e IAF diminuido desenvolverem mordida aberta anterior, assim como os de GoGn-SN diminuído e IAF aumentado desenvolverem sobremordida exagerada $3,4,16,20,21,28,30,38,39,40$. É interessante notar que as avaliações feitas entre as medidas esqueléticas (GoGn-SN e IAF) apresentaram uma forte correlação. Porém, estas medidas mostraram-se

Tabela 2 - Coeficientes de correlação de Pearson e Spearman para as medidas dos indivíduos portadores de má oclusão.

\begin{tabular}{cccc}
\hline correlação de Pearson & GoGn-SN & IAF & sobremordida \\
\hline GoGn-SN & 1,000 & $-0,811^{* *}$ & $-0,293^{*}$ \\
IAF & $-0,811^{* *}$ & 1,000 & $0,291^{*}$ \\
sobremordida & $-0,293^{*}$ & $0,291^{*}$ & 1,000 \\
\hline correlação de Spearman & GoGn-SN & IAF & sobremordida \\
\hline GoGn-SN & 1,000 & $-0,781^{* *}$ & $-0,270^{*}$ \\
IAF & $-0,781^{* *}$ & 1,000 & $0,285^{*}$ \\
sobremordida & $-0,270^{*}$ & $0,285^{*}$ & 1,000 \\
\hline
\end{tabular}

* Significância em nível de 5\%.

** Significância em nível de 1\%. fracamente correlacionadas com a sobremordida, que é uma medida dentária. Isto se deve, provavelmente, ao mecanismo dentoalveolar compensatório dos incisivos, que foi constatado na maioria dos indivíduos deste grupo, e que impede, muitas vezes, o aparecimento de uma mordida aberta ou sobremordida exagerada, mesmo em pacientes com características esqueléticas que predisponham a uma dessas má oclusões.

Ao se correlacionar as três medidas simultaneamente, observou-se que, em $69 \%$ dos traçados dos indivíduos portadores de má oclusão, não houve coerência entre os valores dessas medidas (Gráf. $3)$. Isto se deve ao fato de que, nesse grupo, existem pacientes com tipos faciais extremos, ou seja, dolicocefálicos típicos (GoGn-SN aumentado e IAF reduzido) com mordida aberta, e braquicefálicos típicos (GoGn-SN reduzido e IAF aumentado) com sobremordida profunda. Esses pacientes seriam responsáveis pela correlação coerente com a literatura, porém se apresentavam em minoria, o que justifica o resultado encontrado.

Coben ${ }^{10}$; Lai, Ghosh e Nanda ${ }^{25}$ chamaram a atenção de que muitos trabalhos descreviam a morfologia e o desenvolvimento da face humana em termos gerais. Todavia, tal informação é de utilidade clínica limitada quando se lida com a variação individual. A identificação dos fatores que contribuem para esta variação pode fornecer

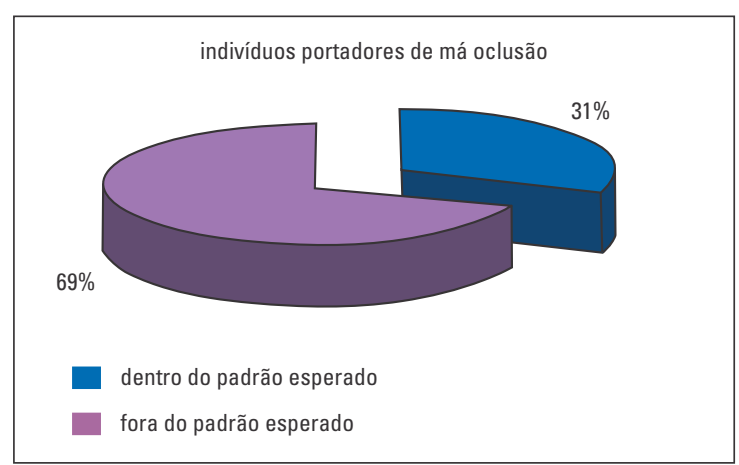

GRÁFICO 3 - Representação gráfica da porcentagem de indivíduos portadores de má oclusão cujo comportamento das medidas se apresentou dentro e fora do padrão citado na literatura. 


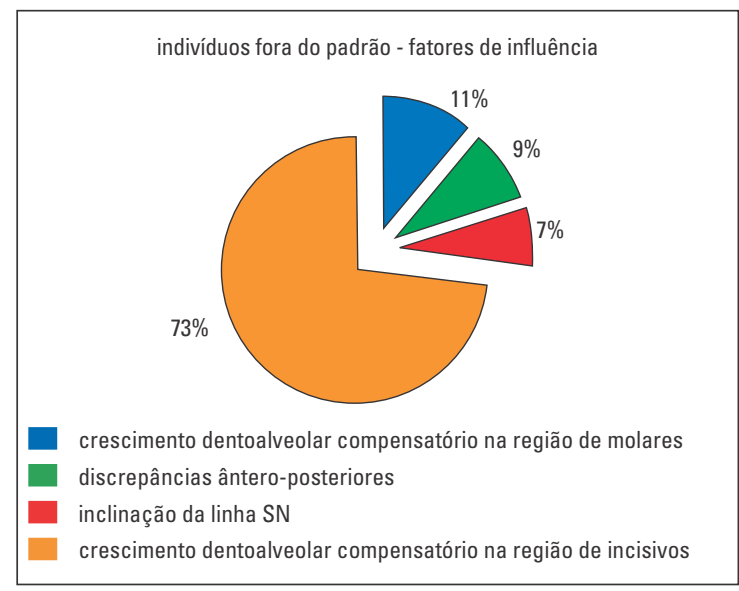

GRÁFICO 4 - Representação gráfica da porcentagem de cada fator que influenciou para que os indivíduos portadores de má oclusão apresentassem o comportamento das medidas fora do padrão citado na literatura.

informações importantes para o diagnóstico clínico de casos específicos, principalmente quando se trata de displasias verticais, devido ao maior grau de dificuldade da terapia ortodôntica.

A análise de medidas que pudessem estar influenciando nos resultados obtidos nos indivíduos com má oclusão, que fugiram ao padrão esperado, mostrou, de acordo com o gráfico 4, que variações dentoalveolares na região de incisivos foram o fator preponderante $(73 \%)$ na compensação das más oclusões, resultado que está de acordo com a literatura pertinente $\mathrm{e}^{14,22,30}$.

Em bem menor escala (11\%), observou-se a influência de compensações dentoalveolares na região de molares. A baixa incidência desse fator pode ser relacionada ao padrão muscular bem definido da face dos indivíduos de face longa e curta, que dificulta, por exemplo, a infra-erupção dos molares nos indivíduos de face longa e a sobreerupção nos de face curta, fatos que poderiam compensar a displasia esquelética ${ }^{33,41}$.

Contudo, inclinações exageradas ou deficientes da linha SN (ângulo SN-PF), que modificariam o valor real do ângulo GoGn-SN, foram encontradas em apenas 7\% dos casos. Em 9\% foram encontradas as discrepâncias ântero-posteriores (ângulo
ANB) que causam, por exemplo, a mordida em topo e impedem a relação de trespasse horizontal normal dos incisivos.

Vale ressaltar, entretanto, a necessidade da realização de estudos adicionais focados na influência de outras estruturas craniofacias, como, por exemplo, o plano palatino e o deslocamento vertical da fossa temporomandibular, no comportamento das medidas verticais. Outro aspecto a ser considerado é o fato de que, nos indivíduos com oclusão excelente, a altura dos processos dentoalveolares dos molares, e não apenas dos incisivos, parece exercer uma grande influência na compensação das displasias esqueléticas verticais. Pode-se sugerir, assim, a realização de novos estudos que relacionem o padrão muscular presente nos diferentes tipos faciais com o crescimento dos processos dentoalveolares dos dentes posteriores, como já antecipado na literatura $^{33,41}$.

\section{CONCLUSÃO}

As medidas esqueléticas GoGn-SN e IAF apresentaram forte correlação entre si, tanto nos pacientes com oclusão excelente quanto nos pacientes com má oclusão. O nível de sobremordida, entretanto, não apresentou correlação com essas medidas em pacientes com oclusão excelente e mostrou-se fracamente correlacionado nos pacientes com má oclusão.

Quando as três medidas foram correlacionadas simultaneamente, a maior parte de ambos os grupos apresentou um comportamento diferente do padrão citado na literatura.

Nos indivíduos portadores de oclusão excelente, o fator que mais contribuiu para que as medidas GoGn-SN, IAF e sobremordida apresentassem entre si um comportamento fora do padrão esperado foi o mecanismo compensatório dentoalveolar na região de incisivos e molares (70\%), seguido de compensação apenas na região de incisivos (15\%) ou molares (15\%). Por outro lado, nos indivíduos portadores de má oclusão, a variação dentoalveolar apenas na região de incisivos (73\%) foi o fator 
preponderante para esse tipo de comportamento entre as medidas. Em bem menor escala, observou-se a influência de compensações dentoalveolares apenas na região de molares (11\%), seguida das discrepâncias ântero-posteriores (9\%) e, por último, alterações na inclinação normal da linha sela-násio (7\%).

\section{AGRADECIMENTOS}

Os autores agradecem à Semp Toshiba, pelo fornecimento dos equipamentos de informática que possibilitaram a realização desta pesquisa.

\title{
Correlative analysis between vertical measurements in individuals with excellent occlusion and malocclusion
}

\begin{abstract}
Introduction: The evaluation of facial vertical proportions is one of the most important features considered by the orthodontist when choosing the appropriate therapy. Meanwhile, when vertical measurements are analyzed separately, one could have an unreliable idea of what the patient's face presents considering vertical development. Aim: The purpose of this research was to perform a correlative analysis between the vertical cephalometric measurements: mandibular plane angle (GoGn-SN), facial height index (FHI) and overbite; to evaluate if the simultaneous correlation of these measurements is in agreement with the results cited in the literature; and to identify the possible factors which could, eventually, lead to different results from those expected. Methods: This study was performed through cephalometric $x$-rays obtained from randomly chosen individuals with excellent occlusion and malocclusion, selected from the Orthodontics Program of the Federal University of Bahia. Results: The skeletal measurements GoGn-SN and FHI showed strong correlation. The overbite level did not showed correlation with these measurements in the excellent occlusion group, and showed weak correlation in the malocclusion group. Only $35 \%$ of the individuals with excellent occlusion showed an expected correlation between the three measurements, while this percentage was of $31 \%$ in the malocclusion group. The remaining individuals showed variations in the incisor dentoalveolar heights as a major factor in compensating the vertical dysplasia, and in the excellent occlusion group it was also found compensations in the molars area. Conclusions: The results indicates that the most part of the groups presented a correlation between the three vertical measurements different from the pattern related in the literature.
\end{abstract}

Key words: Mandibular plane angle. Facial height index. Overbite. Facial vertical dimensions.

\section{REFERÊNCIAS}

1. ANDERSON, D.; POPOVICH, F. Relation of cranial base flexure to cranial form and mandibular position. Am. J. Phys. Anthropol., New York, v. 61, no. 2, p. 181-187, June 1983.

2. ARAÚJO, T. M. de. Cefalometria, conceitos e análises. 1983. Dissertação (Mestrado)-Faculdade de Odontologia, Universidade Federal do Rio de Janeiro, Rio de Janeiro, 1983.

3. BEANE, R. A.; REIMANN, G.; PHILLIPS, C.; TULLOCH, C. A cephalometric comparison of black open-bite subjects and black normals. Angle Orthod., Appleton, v. 73, no. 3, p. 294-300, June 2003.

4. BECKMANN, S. H.; KUITERT, S. R. B.; PRAHL-ANDERSEN, B.; SEGNER, D.; TUINZING, D. B. Alveolar and skeletal dimensions associated with overbite. Am. J. Orthod. Dentofacial Orthop., St. Louis, v. 113, no. 4, p. 443-452, Apr. 1998
5. BECKMANN, S. H.; KUITERT, R. B.; PRAHL-ANDERSEN, B.; SEGNER, D.; TUINZING, D. B. Alveolar and skeletal dimensions associated with lower face height. Am. J. Orthod. Dentofacial Orthop., St. Louis, v. 113, no. 5, p. 498-506, May 1998.

6. BETZENBERGER, D.; RUF, S.; PANCHERZ, H. The compensatory mechanism in high-angle malocclusions: a comparison of subjects in the mixed and permanent dentition. Angle Orthod., Appleton, v. 69, no. 1, p. 27-32, Feb. 1999.

7. BISHARA, S. E.; JAKOBSEN, J. R. Longitudinal changes in three normal facial types. Am. J. Orthod., St. Louis, v. 88 no. 6, p. 466-502, Dec. 1985.

8. BJÖRK, A.; SKEILLER, V. Facial development and tooth eruption: an implant study at the age of puberty. Am. J. Orthod., St. Louis, v. 62, no. 4, p. 339-383, Oct. 1972. 
9. BUSCHANG, P. H.; GANDINI JUNIOR, L. G. Mandibular skeletal growth and modelling between 10 and 15 years of age. Eur. J. Orthod., Oxford, v. 24, no. 1, p. 69-79, Feb. 2002.

10. COBEN, S. E. The integration of facial skeletal variants. A serial cephalometric roentgenographic analysis of craniofacial form and growth. Am. J. Orthod., St. Louis, v. 41, no. 6, p. 407-434, June 1955.

11. DEBERARDINIS, M.; STRETESKY, T.; SINHA, P.; NANDA, R. S Evaluation of the vertical holding appliance in treatment of high-angle patients. Am. J. Orthod. Dentofacial Orthop., St. Louis, v. 117, no. 6, p. 700-705, June 2000

12. FIELDS, H. W.: PROFFIT, W. R.; NIXON, W. L.; PHILLIPS, C. STANEK, E. Facial pattern differences in long-faced children and adults. Am. J. Orthod., St. Louis, v. 85, no. 3, p. 217-223, Mar. 1984.

13. GAO, X.; OTSUKA, R.; ONO, T.; HONDA, E.; SASAKI, T. KURODA, T. Effect of titrated mandibular advancement and jaw opening on the upper airway in non-apneic men: a magnetic resonance imaging and cephalometric study. Am. J. Orthod. Dentofacial Orthop., St. Louis, v. 125, no. 2, p. 191-199, Feb. 2004.

14. HARALABAKIS, N. B.; YIAGTZIS, S. C.; TOUTOUNTZAKIS, N. M. Cephalometric characteristics of open bite in adults: a three-dimensional cephalometric evaluation. Int. J. Adult Orthodon. Orthognath. Surg., Chicago, v. 9, no. 3, p. 223-231, 1994

15. HORN, A. J. Facial height index. Am. J. Orthod. Dentofacial Orthop., St. Louis, v. 102, no. 2, p. 180-186, Aug. 1992.

16. HORN, A. J. L'analyse de Tweed revisitée. Rev. Soc. Bras. Ortod., Rio de Janeiro, v. 3, n. 5, p. 178-187, jan./jun. 1998.

17. ISCAN, H. N.; DINCER, M.; GULTAN, A.; MERAL, O.; TANER-SARISOY, L. Effects of vertical chincap therapy on the mandibular morphology in open-bite patients. Am. J. Orthod. Dentofacial Orthop., St. Louis, v. 122, no. 5, p. 506-511, Nov. 2002.

18. JACOBSON, A. The "Wits" appraisal of jaw disharmony. Am. J. Orthod. Dentofacial Orthop., St. Louis, v. 124, no. 5 , p. 470-479, Nov. 2003.

19. JANSON, G. R. P.; METAXAS, A., WOODSIDE, D. G. Variation in maxillary and mandibular molar and incisor vertical dimension in 12-year-old subjects with excess, normal, and short lower anterior face height. Am. J. Orthod. Dentofacial Orthop., St. Louis, v. 106, no. 4, p. 409-418, Oct. 1994

20. KARLSEN, A. T. Craniofacial characteristics in children with Angle Class II-1 malocclusion combined with extreme deepbite. Angle Orthod., Appleton, v. 64, no. 2, p. 123-130, 1994.

21. KARLSEN, A. T. Craniofacial morphology in children with Angle Class II-1 malocclusion with and without deepbite. Angle Orthod., Appleton, v. 64, no. 6, p. 437-446, 1994

22. KARLSEN, A. T. Association between facial height development and mandibular growth rotation in low and high MP-SN angle faces: a longitudinal study. Angle Orthod. Appleton, v. 67, no. 2, p. 103-110, 1997.

23. KIM, Y. H. Overbite depth indicator with particular reference to anterior open-bite. Am. J. Orthod., St. Louis, v. 65, no. 6, p. 586-664, June 1974.

24. KLUEMPER, G. T.; SPALDING, P. M. Realities of craniofacial growth modification. Atlas Oral Maxillofac. Surg. Clin. North Am., Philadelphia, v. 9, no. 1, p. 23-51, Mar. 2001.
25. LAl, J.: GHOSH, J.: NANDA, R. S. Effects of orthodontic therapy on the facial profile in long and short vertical facial patterns. Am. J. Orthod. Dentofacial Orthop., St. Louis, v. 118, no. 5, p. 505-513, Nov. 2000.

26. MARCHIORO, E. M. Tamanho e localização da mandíbula nos casos de Classe II, $1^{\text {a }}$ divisão comparados a oclusão excelente: adultos não tratados ortodonticamente. 1991. Dissertação (Mestrado)-Faculdade de Odontologia, Universidade Federal do Rio de Janeiro, Rio de Janeiro, 1991.

27. MCNAMARA JR., J. A.; BRUDOM, W. L. Orthodontics and Dentofacial Orthopedics. Michigan: Needham, 2001.

28. MERRIFIELD, L. L.; KLONTZ, H. A.; VADEN, J. L. Differential diagnostic analysis system. Am. J. Orthod. Dentofacial Orthop., St. Louis, v. 106, no. 6, p. 641-648, Dec. 1994.

29. NANDA, S. K. Patterns of vertical growth in the face. Am. J. Orthod. Dentofacial Orthop., St. Louis, v. 93, no. 2, p. 103-116, Feb. 1988

30. NAUMANN, S. A.; BEHRENTS, R. G.; BUSCHANG, P. H. Vertical components of overbite change: a mathematical model. Am. J. Orthod. Dentofacial Orthop., St. Louis, v. 117, no. 4, p. 486-495, Apr. 2000

31. NIELSEN, L. Vertical malocclusions: etiology, development, diagnosis and some aspects of treatment. Angle Orthod., Appleton, v. 61, no. 4, p. 247-260, Apr. 1991.

32. PEARSON, L. E. Vertical control in fully-banded orthodontic treatment. Angle Orthod., Appleton, v. 56, no. 2, p. 205-224, July 1986.

33. PROFFIT, W.; FIELDS, H.: NIXON, W. Occlusal forces in normal and long-face adults. J. Dent. Res., Alexandria, v. 62, no. 5 p. 566-571, May 1983

34. PROFFIT, W. R. Ortodontia contemporânea. 2. ed. Rio de Janeiro: Guanabara Koogan, 1995.

35. SANKEY, W. L.; BUSCHANG, P. H.; ENGLISH, J.; OWEN, A. H. 3rd. Early treatment of vertical skeletal dysplasia: the hyperdivergent phenotype. Am. J. Orthod. Dentofacial Orthop., St. Louis, v. 118, no. 3, p. 317-327, Sept. 2000.

36. SINCLAIR, P. M.; LITTLE, R. M. Dentofacial maturation of untreated normals. Am. J. Orthod., St. Louis, v. 88, no. 2, p. 146-156, Aug. 1985 .

37. STEINER, C. C. Cephalometrics for you and me. Am. J. Orthod., St. Louis, v. 39 no. 10, p. 729-755, Oct. 1953

38. SUGAWARA, J.; BAIK, U. B.; UMEMORI, M.; TAKAHASHI, I.; NAGASAKA, H.; KAWAMURA, H.; MITANI, H. Treatment and posttreatment dentoalveolar changes following intrusion of mandibular molars with application of a skeletal anchorage system (SAS) for open bite correction. Int. J. Adult Orthodon. Orthognath. Surg., Chicago, v. 17, no. 4, p. 243-253, 2002.

39. TSAI, H. H. Cephalometric studies of children with long and short faces. J. Clin. Pediatr. Dent., Birmingham, v. 25, no. 1, p. 23-28, Fall 2000.

40. TSANG, W. M.; CHEUNG, L. K.; SAMMAN, N. Cephalometric characteristics of anterior open bite in a southern Chinese population. Am. J. Orthod. Dentofacial Orthop., St. Louis, v. 113 , no. 2, p. 165-172, Feb. 1998.

41. VAN SPRONSEN, P. H.; WEIJS, W. A.; VALK, J.; PRAHLANDERSEN, B.; VAN GINKEL, F. C. A comparison of jaw muscle cross-sections of long-face and normal adults. J. Dent. Res., Chicago, v. 71, no. 6, p. 1279-1285, June 1992
Endereço para correspondência

Maridélia Machado Mateus Damico

Av. Araújo Pinho, 62, $7^{\circ}$ andar - Canela

CEP: 40.110-150 - Salvador / BA

E-mail: maridamico@uol.com.br 